\title{
Produção Interdisciplinar de Conhecimento Científico no Brasil: temas ambientais
}

Tatiana de P. A. Maranhão ${ }^{1}$

Resumo: A importância dos temas ambientais parece ser compartilhada por diversos discursos políticos internacionais. Apesar desse aparente consenso, países adotam estratégias diferentes de investimentos no desenvolvimento científico e tecnológico, produzido de modo desigual e concentrado. Ademais, questões ambientais constituem objetos de pesquisa extremamente complexos, que necessitam de pesquisas interdisciplinares. No Brasil, houve uma reorientação temática dos investimentos em P\&D, o que poderia incrementar a pesquisa interdisciplinar, mas que, de fato, demonstra a dificuldade de se superar a divisão de campos disciplinares. Analisou-se que o número de grupos de pesquisa, linhas de pesquisa, pesquisadores e produção científica cresceu de modo significativo nos censos de 2000 a 2006. Apesar desse crescimento na pesquisa, manteve-se uma concentração temática nos campos disciplinares: as Ciências Humanas e as Ciências Sociais Aplicadas concentraram-se em pesquisas sobre meio ambiente e desenvolvimento sustentável, enquanto as Biológicas, Agrárias e Exatas dedicaram-se majoritariamente à biodiversidade e aos recursos naturais.

Palavras-chave: interdisciplinaridade; produção interdisciplinar de conhecimento; desenvolvimento científico e tecnológico; pesquisa científica.

\section{Introdução}

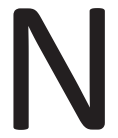

a contemporaneidade de um planeta cujo processo civilizador caracteriza-se por formar redes de interdependências dinâmicas e desiguais entre as sociedades dos indivíduos (Elias, 1994a: 112; 1994b: 264), diversos problemas interpenetram as fronteiras reais das nações e das grandes áreas do conhecimento científico. Problemas mundiais, como a desigualdade socioeconômica e a degradação ambiental (Shiva, 2004: 163-164; Latour, 2002: 15-16), transformados em problemas científicos, transcendem os limites circunscritos pela organização disciplinar do conhecimento (Bursztyn \& Sayago, 2006: 104).

Ocorre que, ao longo da história, com a divisão e a especialização do trabalho
Recebimento:

08.2008

Aprovado:

07.2010

1. Cientista política, mestre em Ciência Política e doutora em Sociologia pela Universidade de Brasília. Pesquisa o fomento público e a produção do conhecimento nas ciências sociais, no âmbito da linha "Educação, Ciência e Tecnologia". tati-maranhao@ uol.com.br 
2. Pode-se citar, como um outro exemplo de esforços coletivos para lidar com fenômenos mundiais complexos, a criação de organismos internacionais organizações nãogovernamentais $\mathrm{e}$ movimentos sociais. Ilustra a preocupação dos organismos internacionais um estudo de Long (2000), que caracteriza a trajetória da Organisation de coopération et de développement économiques - OCDE com relação às questões ambientais internacionais, em mais de três décadas. Segundo Long, houve um momento inicial em que havia pouca competição entre organismos internacionais. Porém, na arena internacional contemporânea, existem diversas instituições com programas ambientais, o que gerou compreensões diferentes, ações multi-institucionais não articuladas em âmbito mundial e uma fragmentação dos esforços de compreensão das questões ambientais. Ademais, identificaram-se algumas questões-chave para o sucesso dos empreendimentos da OCDE, tais como (1) "participação de Ministros do Meio Ambiente de diferentes países e ações transversais entre eles" (Long, 2000: 131132).
(Herrera, 1984: 60-67; Bursztyn, 2005a: 59-76) e com a divisão dos saberes em disciplinas baseadas numa lógica racionalista (Bartholo, 1984:71), houve uma ênfase na separação do ser humano em relação à natureza, na medida em que este pretendeu controlá-la e transformá-la por meio da ciência, da política, da economia. Atualmente, as externalidades, os riscos e a incerteza de um contexto de relações mundializadas, num tempo-espaço que ocorre em fluxos dinâmicos e velozes (Castells, 2005; Beck, Giddens \& Lash, 1997; Beck, 2002), reduzem a crença humana acerca das possibilidades de ação e de compreensão sobre a Natureza, assim como se reduz a capacidade desta de renovação.

A prática interdisciplinar de pesquisa ocorre em meio a diversos contextos no âmbito da história e consiste num empreendimento coletivo (e controverso) para conhecer as realidades ${ }^{2}$. Tal contexto, caracterizado pela complexidade (Baumgarten, 2006; Floriani, 2006; Zellmer et al, 2006), indica a necessidade de novas explicações científicas multidimensionais de médio e longo alcance (Baumgarten, 2006: 16-17), principalmente quando se investigam objetos complexos, que escapam às explicações de uma única disciplina (Floriani, 2006: 72).

Diante dos desafios da contemporaneidade, o presente artigo pretende analisar certos aspectos da pesquisa interdisciplinar (Sobral, 2006a; 2006b), com base em informações sobre programas de pós-graduação interdisciplinares e dados dos quatro Censos realizados pelo Conselho Nacional de Desenvolvimento Científico e Tecnológico - CNPq, em 2000, 2002, 2004 e 2006, sobre o diretório dos Grupos de Pesquisa. Uma vez que saberes disciplinares isolados são insuficientes para a análise (e solução) de problemas ambientais complexos, há que se enfrentar desafios e transcender os limites do campo científico e da organização disciplinar do conhecimento. Portanto, quais são os significados da interdisciplinaridade na produção do conhecimento científico contemporâneo?

\section{Significados da interdisciplinaridade}

A concepção utilizada pelo presente artigo está de acordo com a definição estabelecida por Lélé \& Norgaard:

(...) o termo interdisciplinaridade pode ser usado para descrever todos os tipos de cruzamentos entre as disciplinas, desfazendo as sutis diferenças entre multi-, inter- e trans-, levantadas em discussões mais elaboradas sobre o assunto (2005: 967).

Tal posicionamento considera que disciplinas são artefatos acadêmico-administrativos e que, para analisar problemas complexos como a questão ambiental, é preciso pensar nas comunidades científicas sem se restringir aos saberes compartimentalizados em disciplinas curriculares (Lélé \& Norgaard, 2005: 972). 
Pressupõe-se, portanto, que "é impossível a construção de uma única, absoluta e geral teoria da interdisciplinaridade" (Fazenda, 1994: 13), principalmente pelo fato dessa interdisciplinaridade se constituir na prática, ou seja, ser "mais processo do que produto" (Fazenda, 1994: 25). "O significado de interdisciplinaridade evidencia um cruzamento de saberes disciplinares no campo científico e um esforço organizado de coordenação, cooperação e comunicação menos assimétrica" (Teixeira, 2004: 64).

Dadas as condições sociocognitivas, políticas e econômicas que podem influenciar a produção do conhecimento científico, a aproximação dos saberes propicia um cenário em que, necessariamente, aqueles que participam dessa produção podem "retraduzir tais influências a partir de seu habitus no campo científico" (Bourdieu, 2001; Bourdieu \& Wacquant, 1992). Com esse retorno reflexivo (Bourdieu, 2003), a prática científica encontra-se permeada pela diferente compreensão que os cientistas fazem da realidade (Maranhão, 2005; Sobral, 2006b; Sobral \& Maranhão, 2008).

Outros pesquisadores também sugeriram, de diferente forma, uma incorporação da agenda pública na agenda científica. No novo modo de produção do conhecimento, o Modo 2, existem novas práticas, suficientemente diferentes na linguagem, nos métodos, nos conceitos (Gibbons et al, 1994). Ele se caracteriza pela interdisciplinariedade, empirismo, heterogeneidade institucional (empresas, governo, academia) e responsabilidade social do conhecimento (diferente do Modo 1, que seria o conhecimento tradicional, disciplinar, conhecimento teórico-acadêmico, homogêneo, hierárquico).

Além de reunir vários especialistas de diferentes áreas do conhecimento, com diversos saberes especializados, a prática interdisciplinar de produção de conhecimento possibilita uma evolução da aplicação de conceitos e métodos de diferentes áreas, gerando algo que não se encaixa perfeitamente em nenhuma delas. Ou seja, a interdisciplinaridade surge no contexto da aplicação e necessita de difusão e comunicação de resultados parciais ao longo de um processo dinâmico de pesquisa.

Nesse contexto, várias instituições produzem conhecimento de forma interdependente, sendo governos, empresas, universidades, entre outros, locus potenciais para o desenvolvimento da ciência e da tecnologia (Sobral, 2004; 2006b). Também se percebe um aumento de consciência social - sobre meio ambiente, saúde, entre outros temas - que pode levar a um interesse maior da sociedade sobre as linhas temáticas das pesquisas, influenciando-as desde a escolha do objeto. Assim, há uma sensibilidade do cientista para com essa participação social e os problemas que trazem para a pesquisa. 
Outro aspecto do Modo 2 é o controle de qualidade: não se avalia o conhecimento produzido apenas pelos pares; há critérios adicionais, como interesses sociais, econômicos e políticos. A contribuição é de interesse do mercado? Qual será o custo efetivo da nova tecnologia? Ela é poluente? É culturalmente viável? Há um amplo conjunto de critérios e de questões que refletem a composição ampliada no novo modo de produção.

Nowotny et al (2001), em a Nova Produção do Conhecimento, caracteriza a Sociedade, bem como os fatores relacionados à produção da Ciência. O objetivo do livro consiste em analisar a relação dinâmica, 'íntima', interativa e coevolucionista entre a Ciência e a Sociedade, que ocorre dentro de um contexto histórico. Num cenário de complexidade e incerteza, quando um paradigma propõe explicações rígidas e dualistas, ele tende a perder sua capacidade explicativa e espaço no discurso da comunidade científica.

O mesmo ocorre quando se assume que a comunicação e as relações entre Ciência e Sociedade fluem em via de mão única. Na verdade, uma nova perspectiva capaz de considerar as realidades sociais e as práticas científicas ofereceria explicações mais completas sobre essa relação. Os autores concordam sobre a afirmação de que Sociedade, Cultura e Economia deixam de ser distintas e distinguíveis; tornam-se arenas interdependentes e transgressoras entre si e heterogêneas em si, ao longo do tempo (Nowotny et al, 2001: 1-3; Knorr-Cetina, 1982).

No Brasil, a produção de conhecimento científico ocorre no âmbito de um modelo misto de desenvolvimento científico e tecnológico (Sobral e Trigueiro, 1994).

Esse modelo procura associar a lógica do campo científico, ou seja, as demandas da própria evolução da ciência às demandas econômicas e sociais, isto é, permite a articulação do mercado científico ao mercado econômico e social, ao mesmo tempo em que possibilita a fluidez de fronteiras entre as disciplinas. Porém, esse modelo pode reconhecer as diferenças entre as áreas, umas mais científicas e outras mais aplicadas e tecnológicas, ou ainda umas mais articuladas às demandas sociais e outras às demandas econômicas do setor produtivo. (Sobral, 2004: 11)

Como analisado anteriormente, percebe-se que as fronteiras disciplinares circunscrevem e, de certa forma, restringem o âmbito da ação científica. Não se pretende afirmar um fim da organização disciplinar de conhecimento: de fato, há que se conhecer em profundidade determinada área para que se possa efetivamente colaborar para com a solução interdisciplinar de problemas complexos. Mais do que considerar a interdisciplinaridade como princípio de organização do trabalho científico, o que se afirma é a "necessidade real de encontrar novas explicações para as quais as disciplinas isoladamente são insuficientes" 
(Epstein, 2003). A interdisciplinaridade, então, surge como processo na produção de conhecimento para explicar fenômenos complexos e interdependentes, como os problemas ambientais, cada vez mais relacionados à violência, às guerras e ao desenvolvimento (Morin, 1993: 421; Gorbachev, 2003: 25).

Com isso, como se caracteriza a produção de conhecimento científico no Brasil relacionado à questão ambiental? É possível observar a prática interdisciplinar por meio dos dados disponíveis sobre os programas de pós-graduação multidisciplinares e sobre os grupos de pesquisa com temáticas ambientais?

\section{Questões Ambientais e Interdisciplinaridade na Pós-Graduação}

Inserida nesse cenário global, a produção interdisciplinar de pesquisa no Brasil apresenta certas especificidades temáticas no campo científico. Em 2001, um dos desafios estratégicos estabelecidos como prioridade para o desenvolvimento de uma política nacional de Ciência, Tecnologia e Inovação consistiu no tema "Gestão do Meio Ambiente" (MCT, 2001). Partiu-se do pressuposto de que "a vida humana não pode ser entendida de modo dissociado dos processos naturais" (MCT, 2001: 173), algo presente no pressuposto de Norbert Elias (1994a; 1994b). Tal documento governamental afirma que

\section{A humanidade preserva uma dependência ancestral em relação aos ecos- sistemas que a rodeiam e continua a se valer de outros organismos e de recursos naturais para sua alimentação, atividade econômica e sobrevi- vência em geral. (MCT, 2001: 173)}

Essa afirmativa da sustentabilidade ${ }^{3}$ - a garantia "às futuras gerações de um estoque de capital econômico, humano e ambiental pelo menos equivalente ao atual" (Ibidem), acima de tudo, como estratégia de sobrevivência - é compartilhada, de certa forma, por cientistas e intelectuais, e diversos atores políticos. No caso brasileiro, Fernanda Sobral analisa a inclusão da dimensão social na reorientação dos investimentos públicos federais - especificamente, no PPA20042007 em Ciência e Tecnologia, antes com foco central na dimensão econômica:

O segundo eixo inclui os objetivos estratégicos nacionais, nos quais são enfatizados o programa espacial, o programa nuclear, a Amazônia, o Cerrado, o Pantanal, o Semi-Árido e a plataforma marítima brasileira. O terceiro eixo é o da inclusão social que apóia programas e ações voltadas para a difusão científica e tecnológica, como também a criação de centros vocacionais tecnológicos (CVTs), cujo objetivo é garantir emprego e renda nas regiões mais pobres do país. Nesse eixo, alguns programas se destacam como o de Tecnologias Sociais, Arranjos Produtivos Locais, Biodiesel, Tecnologias de Habitação, Saneamento Básico, Incubadoras em Cooperativas Populares,
3. De acordo com Sachs, existem cinco pilares do desenvolvimento sustentável: (a) social; (b) ambiental; (c) territorial; (d) econômico e (e) político (Sachs, 2004:15-16). Ademais, vide Veiga (2005), Nelissen et al (1997) e Viola (1998). 
Saúde, Desenvolvimento Local, Segurança Nutricional e Alimentar e Inclusão Digital. (Sobral, 2006a: 4)

Sobral evidencia que, mesmo com a reorientação temática dos investimentos em ciência e tecnologia, os editais temáticos financiados pelos Fundos Setoriais "continuam concentrados em determinadas áreas do conhecimento, expressando a dificuldade de implementação efetiva da interdisciplinaridade" (Sobral, 2006a: 19). Tal dificuldade de implementação da prática interdisciplinar também surge na análise de Sobral no caso da política de pós-graduação no período de 1987 a 2003, o que a faz identificar

(...) a necessidade de se pensar a política de pós-graduação de uma forma menos homogênea e mais diversificada, visando incorporar perfis diferentes tanto de disciplinas como de temas multidisciplinares, respeitando também vocações regionais e institucionais. (2006b: 170)

O que se percebe quando a "causa ambiental emerge como foco de interesse no mundo acadêmico" (Bursztyn, 2004: 68) é que o sistema universitário público brasileiro encontra-se em crise, para alguns, uma "crise de confiança epistemológica" (Santos, 1999: 282-283). Até 1985, existiam 54.000 pesquisadores, vinculados a 36.000 projetos. Destes, um total de 580 tratava sobre "meio ambiente e recursos naturais". As áreas do conhecimento que dominavam tais projetos relacionavam-se principalmente às Engenharias, à Biologia e à Química, que correspondiam a 95,9\% do total (Bursztyn, 2004: 68). "Observa-se, dessa forma, a existência de um 'duplo movimento': há, simultaneamente, um destaque maior para a questão ambiental como objeto de pesquisa e um maior número de programas interdisciplinares na Pós-Graduação" (Bursztyn, 2004: 70).

Com esse duplo movimento, as instituições científicas e tecnológicas (universidades, centros de pesquisa) e os órgãos governamentais de fomento têm procurado aperfeiçoar suas referências institucionais sobre a pós-graduação. Nota-se que o CNPq define oito grandes áreas do conhecimento, enquanto a CAPES inclui uma nona área, intitulada "Outras" em 2006 e redefinida como "Multidisciplinar" em 2008. Nessa grande área, encontravam-se programas de pós-graduação com a definição de área de conhecimento "Multidisciplinar" (que, em 2008, passam a ser definidos como área de conhecimento "Interdisciplinar"). Em 2006, havia um total de 189 cursos de pós-graduação, sendo 115 de Mestrado, 38 de especialização e 36 de Doutorado reconhecidos (CAPES, 2008). Destes 189 cursos, apenas seis possuíam avaliação nota 5 e não há nenhum curso avaliado como 6 ou 7, o que pode indicar uma certa resistência na cultura institucional-disciplinar das universidades e/ ou a necessidade de estabelecer critérios específicos de avaliação de programas interdisciplinares. 
Em 2008, tal situação sofreu alterações: de um total de 240 cursos de pós-graduação designados na grande área "multidisciplinar", 190 são programas e cursos da área de conhecimento "interdisciplinar", sendo que 96 oferecem Mestrados, 9 Doutorados, 48 Mestrados e Doutorados e 73 Mestrados profissionais reconhecidos (CAPES, 2008). Dentre esses, 17 receberam avaliação nota 5, mais do que o dobro de programas interdisciplinares com essa nota na avaliação anterior da CAPES, o que indica um aumento significativo na qualidade (e quantidade) de programas reconhecidos. Apesar disso, ainda não há nenhum curso avaliado com notas 6 ou 7, o que reforça a hipótese de resistência na cultura institucional/disciplinar das universidades e de contínuo aprimoramento dos sistemas de avaliação.

Além da questão da avaliação e da qualidade desses programas, é interessante destacar que existe uma área de conhecimento intitulada "Ecologia e Meio Ambiente", com 25 programas de pós-graduação, sendo 3 deles avaliados com nota 6 . Mesmo assim, cerca de um terço de todos os programas interdisciplinares tratam de alguma questão relacionada à pesquisa ambiental e, em sua maioria, mesclam algumas das quatro temáticas aqui pesquisadas - Meio Ambiente, Desenvolvimento Sustentável, Biodiversidade e Recursos Naturais e outros focos de interesse, tais como Saúde, Energia, Direito, Sociedade, Gestão e Tecnologia (Quadro 1).

Quadro 1

Programas de Pós-Graduação relacionados a temáticas ambientais

\begin{tabular}{|l|l|}
\hline PROGRAMAS INTERDISCIPLINARES & NÍVEL (ICT) \\
\hline $\begin{array}{l}\text { AMBIENTE CONSTRUÍDO E PATRIMÔNIO } \\
\text { SUSTENTÁVEL }\end{array}$ & 1 MESTRADO (UFMG) \\
\hline AMBIENTE E DESENVOLVIMENTO & 1 MESTRADO (UNIVATES) \\
\hline AMBIENTE E SOCIEDADE & 1 DOUTORADO (UNICAMP) \\
\hline BIOÉTICA & 1 DOUTORADO (UNB); \\
& 2 MESTRADOS (UNB; CUSC) \\
\hline CIÊNCIA AMBIENTAL & 1 DOUTORADO (USP); \\
& 2 MESTRADOS (UFF; USP) \\
\hline CIÊNCIA E TECNOLOGIA AMBIENTAL & 1 MESTRADO (UNIVALI) \\
\hline CIÊNCIAS AMBIENTAIS & 1 DOUTORADO (UFG); \\
& 5 MESTRADOS (UNEMAT; UFPA; UNESC; \\
& UNOCHAPECÓ; UFT); \\
& 1 MESTRADO PROFISSIONAL (UNITAU) \\
\hline
\end{tabular}


(continuação Quadro 1)

\begin{tabular}{|c|c|}
\hline CIÊNCIAS AMBIENTAIS E SAÚDE & 1 MESTRADO (UCGO) \\
\hline $\begin{array}{l}\text { CIÊNCIAS DO AMBIENTE E SUSTENTABILI- } \\
\text { DADE NA AMAZÔNIA }\end{array}$ & 1 MESTRADO (UFAM) \\
\hline $\begin{array}{l}\text { DESENVOLVIMENTO REGIONAL E MEIO } \\
\text { AMBIENTE }\end{array}$ & 3 MESTRADOS (UESC; UNIR; UNIARA) \\
\hline DESENVOLVIMENTO E MEIO AMBIENTE & $\begin{array}{l}6 \text { MESTRADOS (UFC; UFPB; UFPE; FUFPI; } \\
\text { UFRN; FUFSE) }\end{array}$ \\
\hline DESENVOLVIMENTO RURAL & 1 DOUTORADO E 1 MESTRADO (UFRGS) \\
\hline DESENVOLVIMENTO SUSTENTÁVEL & $\begin{array}{l}1 \text { DOUTORADO; } 1 \text { MESTRADO; } 1 \text { MES- } \\
\text { TRADO PROFISSIONAL (UNB) }\end{array}$ \\
\hline $\begin{array}{l}\text { DESENVOLVIMENTO SUSTENTÁVEL DO } \\
\text { TRÓPICO ÚMIDO }\end{array}$ & 1 DOUTORADO E 1 MESTRADO (UFPA) \\
\hline $\begin{array}{l}\text { DIREITO AMBIENTAL E POLÍTICAS PÚBLI- } \\
\text { CAS }\end{array}$ & 1 MESTRADO (UNIFAP) \\
\hline ECOLOGIA APLICADA & $\begin{array}{l}1 \text { DOUTORADO E } 1 \text { MESTRADO (USP/ } \\
\text { ESALQ) }\end{array}$ \\
\hline ECOLOGIA E PRODUÇÃO SUSTENTÁVEL & 1 MESTRADO (UCGO) \\
\hline ENERGIA E AMBIENTE & 1 DOUTORADO (UFBA) \\
\hline $\begin{array}{l}\text { ENGENHARIA: ENERGIA, AMBIENTE E } \\
\text { MATERIAIS }\end{array}$ & 1 MESTRADO PROFISSIONAL (ULBRA) \\
\hline FÍSICA AMBIENTAL & 1 DOUTORADO E 1 MESTRADO (UFMT) \\
\hline $\begin{array}{l}\text { GERENCIAMENTO E TECNOLOGIA AM- } \\
\text { BIENTAL NO PROCESSO PRODUTIVO }\end{array}$ & 1 MESTRADO PROFISSIONAL (UFBA) \\
\hline GESTÃO AMBIENTAL & 1 MESTRADO PROFISSIONAL (UNICENP) \\
\hline $\begin{array}{l}\text { GESTÃO INTEGRADA EM SAÚDE DO TRA- } \\
\text { BALHO E MEIO AMBIENTE }\end{array}$ & 1 MESTRADO PROFISSIONAL (SENAC) \\
\hline MEIO AMBIENTE & 1 DOUTORADO (UERJ) \\
\hline MEIO AMBIENTE E DESENVOLVIMENTO & 1 DOUTORADO (UFPR) \\
\hline $\begin{array}{l}\text { MEIO AMBIENTE E DESENVOLVIMENTO } \\
\text { REGIONAL }\end{array}$ & 1 MESTRADO (UNIDERP) \\
\hline MEIO AMBIENTE E RECURSOS HÍDRICOS & 1 MESTRADO (UNIFEI) \\
\hline MEIO AMBIENTE E SUSTENTABILIDADE & 1 MESTRADO PROFISSIONAL (UNEC) \\
\hline $\begin{array}{l}\text { MODELAGEM EM CIÊNCIAS DA TERRA E } \\
\text { DO AMBIENTE }\end{array}$ & 1 MESTRADO (UEFS) \\
\hline PLANEJAMENTO E GESTÃO AMBIENTAL & 1 MESTRADO (UCB) \\
\hline QUALIDADE AMBIENTAL & 1 MESTRADO (FEEVALE) \\
\hline RECURSOS HÍDRICOS & 1 MESTRADO (UFMT) \\
\hline
\end{tabular}


(continuação Quadro 1)

\begin{tabular}{|l|l|}
\hline RECURSOS NATURAIS & $\begin{array}{l}1 \text { DOUTORADO (UFCG); } \\
\text { 2 MESTRADOS (UFCG; UFRR) }\end{array}$ \\
\hline SAÚDE E AMBIENTE & 3 MESTRADOS (UFMA; UNIT; UNIVILLE) \\
\hline $\begin{array}{l}\text { SOCIEDADE, TECNOLOGIA E MEIO AM- } \\
\text { BIENTE }\end{array}$ & 1 MESTRADO (UNIEVANGEL) \\
\hline SUSTENTABILIDADE DE ECOSSISTEMAS & 1 MESTRADO (UFMA) \\
\hline $\begin{array}{l}\text { SUSTENTABILIDADE SOCIOECONÔMICA } \\
\text { AMBIENTAL }\end{array}$ & 1 MESTRADO PROFISSIONAL (UFOP) \\
\hline TECNOLOGIA AMBIENTAL & 1 MESTRADO PROFISSIONAL (ITEP; IPT) \\
\hline
\end{tabular}

Fonte: CAPES, 2008b (adaptado)

O sentido desse levantamento reside na possibilidade de se chegar, por meio dos Cadernos de Indicadores do sistema de avaliação da CAPES, aos autores e aos títulos da produção científica e tecnológica vinculados a esses programas interdisciplinares com foco ambiental ${ }^{4}$. Ainda assim, há fortes indícios de que os programas de pós-graduação interdisciplinares são realmente o locus privilegiado da produção interdisciplinar de conhecimento científico no País 5 .

\section{Produção interdisciplinar de pesquisa científica: o Diretório de Grupos de Pesquisa do CNPq}

A fim de ampliar a compreensão sobre "quem produz esse tipo de conhecimento e em que locus isso ocorre" (Sobral, 2006b), identificaram-se os grupos de pesquisa, as linhas de pesquisa, os pesquisadores, os estudantes e a produção existentes no Diretório de Grupos de Pesquisa do sistema Lattes do CNPq que lidam com questões ambientais. Feita uma observação anterior sobre os programas de pós-graduação a partir do sistema de avaliação da CAPES, coube abordar a produção interdisciplinar noutra base de dados, com recorte diferente. Embora os sistemas de avaliação da CAPES e o Lattes do CNPq recebam informações sobre a comunidade científica, recebem-nas por diferentes instrumentos de coleta ${ }^{6}$. Foram procuradas quatro palavras-chave no sistema Lattes/CNPq: meio ambiente, desenvolvimento sustentável, biodiversidade e recursos naturais (Bursztyn, 2004: 69).

O último Censo realizado no Diretório de Grupos de Pesquisa do CNPq ocorreu em 2006, com um total de 21.024 grupos (CNPq, 2008). A fim de verificar a variação na quantidade de grupos de pesquisa, realizou-se um levantamento nesse Diretório a partir da ferramenta de busca textual, restringindo os resultados às ocasiões em que as palavras-chave meio ambiente, desenvolvimento sustentável, biodiversidade e recursos naturais aparecessem apenas em "nome de grupo de pesquisa".

4. Esse levantamento aprofundado exige da pesquisadora mais recursos do que se tem para o presente artigo, mas continua em desenvolvimento até o final de 2008.

5. Compreendendo a produção de conhecimento num modelo misto de desenvolvimento científico e tecnológico, outras arenas de produção ocorrem nas empresas, nos centros de pesquisa públicos e privados. Porém, torna-se viável começar a busca nas bases de dados existentes sobre as universidades, no caso brasileiro.

6. Por exemplo, cada estudante, professor e pesquisador preenche seus dados pessoais no Currículo Lattes. Cada coordenador de pesquisa gerencia os dados de seu grupo e linha de pesquisa. As equipes técnicas dos departamentos nas universidades auxiliam comissões de professores de pósgraduações na coleta de dados para a avaliação da CAPES. 
7. Para o levantamento referente às linhas de pesquisa, fez-se a busca textual no Diretório para os quatro temas, quando estes fossem citados no "nome da linha de pesquisa" e nas "palavras-chave da linha".

De acordo com os Gráficos 1 e 2, houve um crescimento no número absoluto de grupos e de linhas de pesquisa ${ }^{7}$ nos quatro temas, com destaque para os grupos identificados com meio ambiente, que se tornaram 210 em 2006, mais do que a soma dos grupos relacionados à biodiversidade, recursos naturais e desenvolvimento sustentável no mesmo ano.

Gráfico 1 - Grupos de pesquisa por tema ambiental

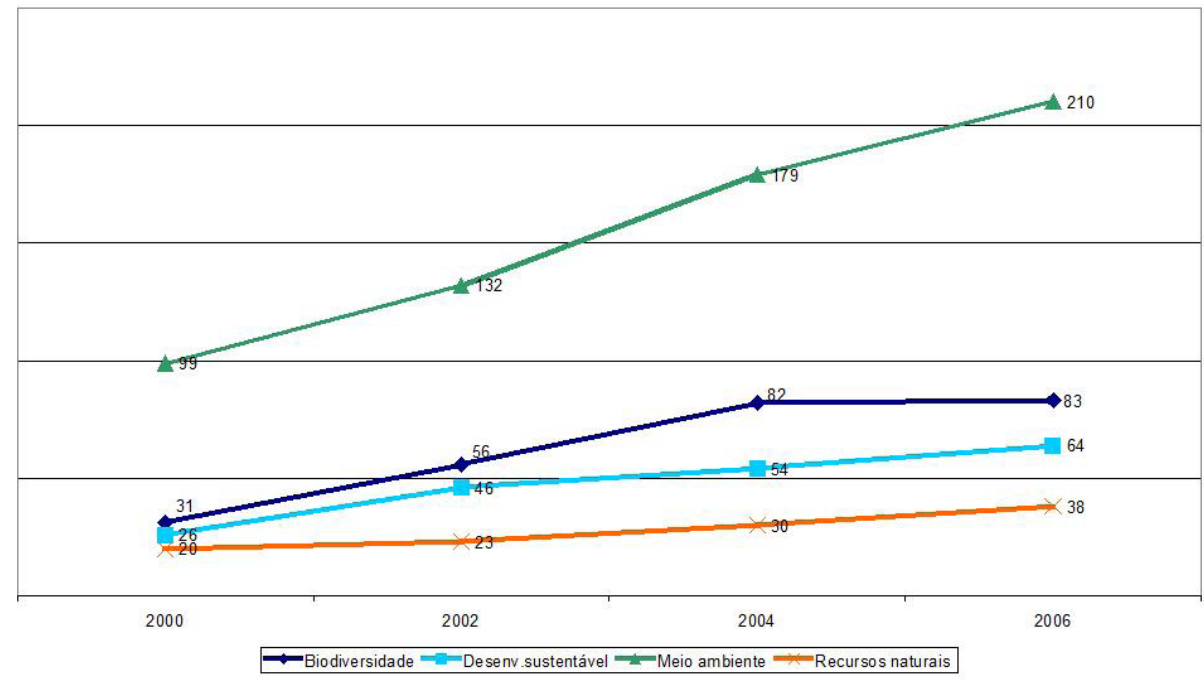

Gráfico 2 - Linhas de pesquisa por tema ambiental

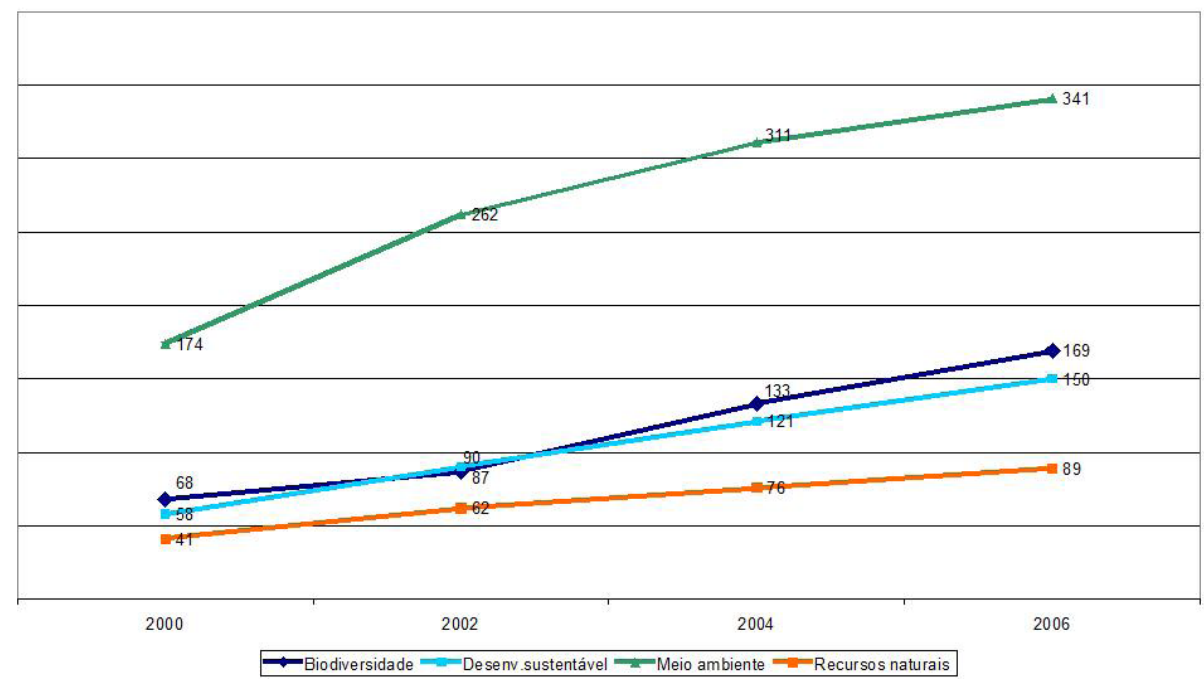


Quanto aos pesquisadores ${ }^{8}$, observou-se um aumento de cerca de 200 pesquisadores, a cada Censo, para cada temática ambiental analisada, exceto Recursos Naturais (Gráfico 3). A produção científica, tecnológica e artística9 sobre biodiversidade, meio ambiente, desenvolvimento sustentável e recursos naturais também foi ampliada no período observado (Gráfico 4).

Gráfico 3 - Pesquisadores por tema ambiental

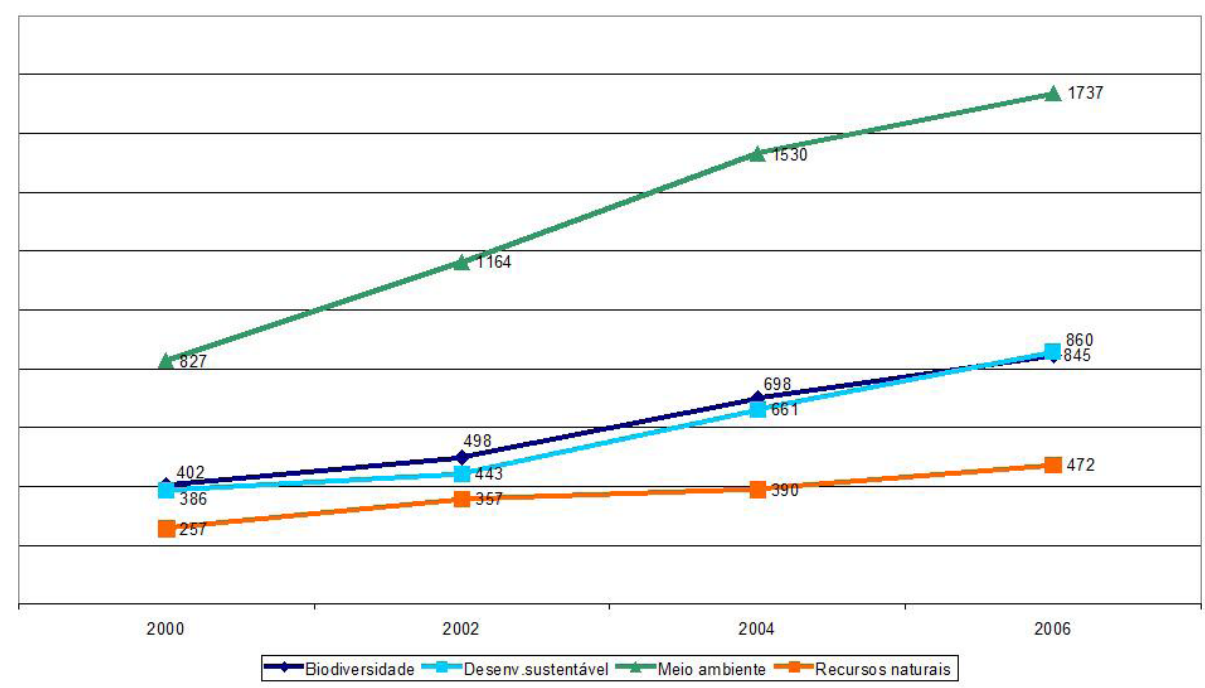

Gráfico 4 - Produção cientifica, tecnológica e artística por tema ambiental

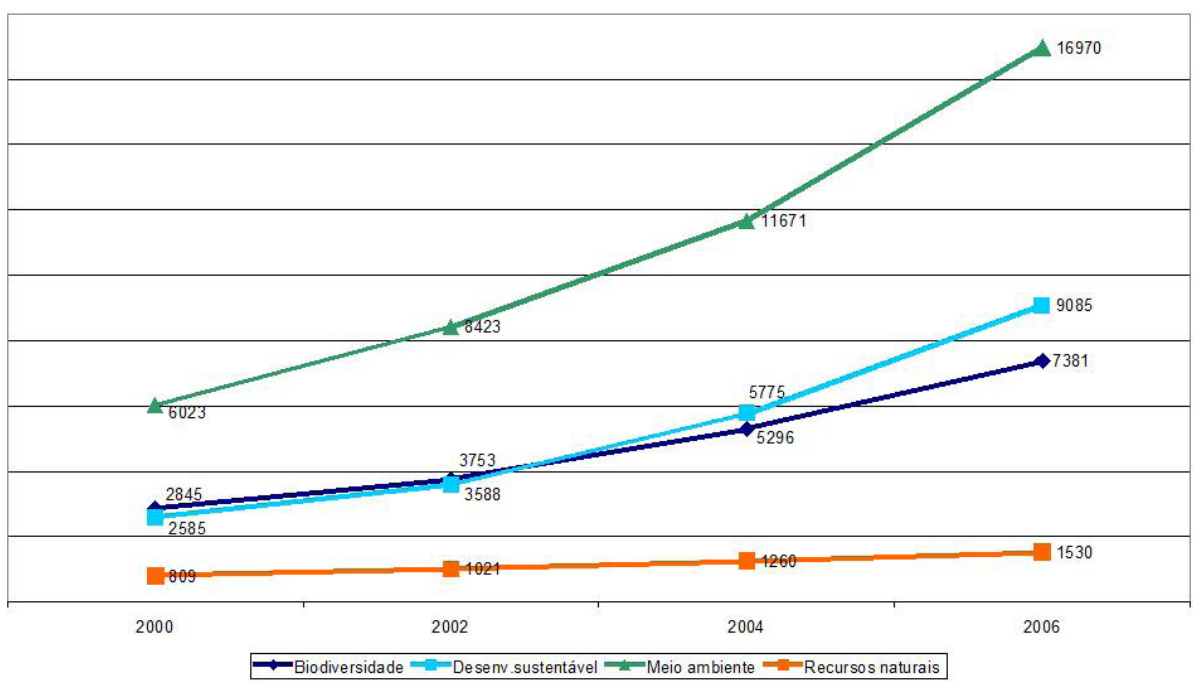

8. Para o levantamento referente aos pesquisadores, fez-se a busca textual no Diretório para os quatro temas, quando estes fossem citados no "nome do grupo", "nome da linha de pesquisa" e nas "palavras-chave da linha".

9. Busca textual restrita a "título da produção CTA" e "palavra-chave da produção". 
10. A produção CTA dá-se pelo somatório de (a) artigos completos de circulação nacional; (b) artigos completos de circulação internacional; (c) trabalhos completos publicados em anais; (d) livros; (e) capítulos de livros; (f) produção técnica; (g) teses e (h) dissertações (CAPES, 2008).

11. O número de estudantes que têm participado de grupos de pesquisa foram levantados a partir da ferramenta "busca textual" no Diretório de Grupos de Pesquisa do CNPq, restrita ao "nome do grupo" para as quatro temáticas ambientais nos quatro Censos. Quantos estudantes vinculam-se aos grupos de pesquisa que possuem o termo "biodiversidade" em sua identificação, por exemplo? Algo que necessita de pesquisas posteriores para ser explicado é o porquê de tal redução.
Gráfico 5 - Estudantes por tema ambiental

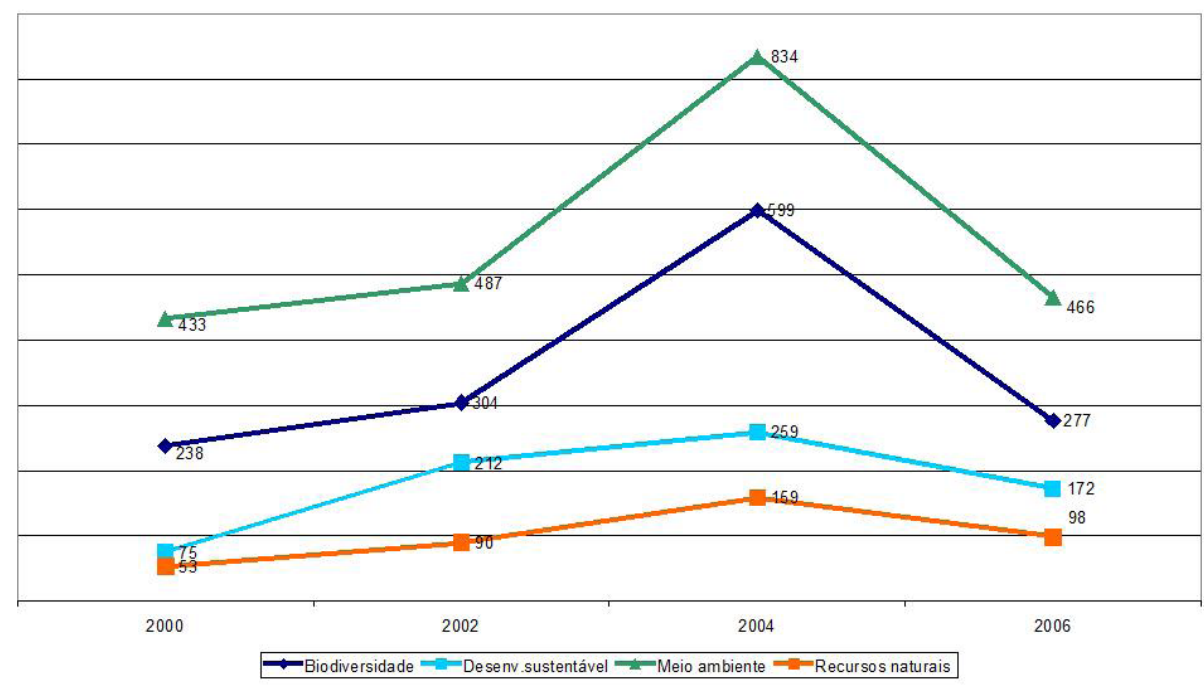

Ao analisar os dados do CNPq de modo desagregado, nos quatro Censos realizados com os grupos de pesquisa do sistema Lattes (2000, 2002, 2004 e 2006), verifica-se um crescimento no número absoluto de grupos de pesquisa, linhas de pesquisa, pesquisadores e da produção científica, tecnológica e artística (produção $C T A^{10}$ ) nos quatro temas ambientais analisados (meio ambiente, desenvolvimento sustentável, biodiversidade e recursos naturais), de 2000 a 2004 (vide Gráficos 1 a 5), alguns com maior e outros com menor intensidade. A única exceção ao movimento crescente foi uma redução do número de alunos vinculados a esses grupos em todas as temáticas ambientais pesquisadas entre o Censo 2004 e o Censo $2006^{11}$.

Apesar desse crescimento absoluto do número de grupos de pesquisa, linhas, pesquisadores e produção CTA observado em todas as temáticas, ao se compararem as taxas de variação entre os censos, verificam-se reduções no percentual de crescimento em algumas dessas categorias, com destaque para a produção científica dos grupos de pesquisa. Isso significa, por exemplo, que, embora tenha havido um crescimento no número absoluto da produção científica (produção CTA), ela cresceu menos no período de 2002 a 2004, quando comparada com o período de 2000 a 2002 em todos os temas analisados. Entretanto, há que se considerar a expansão da Pós-Graduação brasileira nesse período (Martins, 2000), com a inclusão de muitos estudantes e pesquisadores recentes, com produção relativamente restrita em relação aos pesquisadores com mais tempo de acúmulo e de produtividade nos grupos de pesquisa (artigos, livros, produtos técnicos, entre outros). 
Por meio da análise temática relacionada às grandes áreas do conhecimento a que se vinculam grupos de pesquisa cadastrados na base CNPq/LATTES, até fevereiro de 2008 , observa-se uma concentração temática nos grupos de pesquisa que varia de acordo com as grandes áreas do conhecimento (Tabela 1).

Tabela 1 - Temas Ambientais dos Grupos de Pesquisa por Grande Área do Conhecimento

\begin{tabular}{|c|c|c|c|c|c|c|c|c|}
\hline \multirow{3}{*}{$\begin{array}{l}\text { Grande Área do } \\
\text { Conhecimento CNpq }\end{array}$} & \multicolumn{8}{|c|}{ Grupos de Pesquisa CNPq por Temas } \\
\hline & \multicolumn{2}{|c|}{ Meio Ambiente (\%) } & \multicolumn{2}{|c|}{$\begin{array}{l}\text { Desenvolvimento } \\
\text { Sustentável (\%) }\end{array}$} & \multicolumn{2}{|c|}{ Biodiversidade (\%) } & \multicolumn{2}{|c|}{$\begin{array}{c}\text { Recursos Naturais } \\
(\%)\end{array}$} \\
\hline & 2006 & 2008 & 2006 & 2008 & 2006 & 2008 & 2006 & 2008 \\
\hline Ciências Agrárias & 6,9 & 7,6 & 15,8 & 16,1 & 16,2 & 17,0 & 31,7 & 30,8 \\
\hline Ciências Biológicas & 7,2 & 7,3 & 9,3 & 9,2 & 64,9 & 63,0 & 16,0 & 16,1 \\
\hline Ciências da Saúde & 7,0 & 6,1 & 1,9 & 1,4 & 2,4 & 2,2 & 1,2 & 1,0 \\
\hline $\begin{array}{l}\text { Ciências Exatas e da } \\
\text { Terra }\end{array}$ & 16,4 & 15,5 & 5,0 & 5,5 & 7,2 & 8,4 & 19,8 & 17,5 \\
\hline Ciências Humanas & 21,5 & 21,9 & 19,4 & 20,0 & 4,2 & 4,1 & 10,3 & 11,5 \\
\hline $\begin{array}{ll}\text { Ciências } & \text { Sociais } \\
\text { Aplicadas } & \end{array}$ & 24,1 & 24,6 & 37,4 & 37,9 & 4,0 & 4,3 & 15,6 & 16,8 \\
\hline Engenharias & 16,5 & 16,8 & 11,2 & 9,9 & 1,2 & 1,1 & 5,3 & 6,3 \\
\hline $\begin{array}{l}\text { Linguística, Letras e } \\
\text { Artes }\end{array}$ & 0,3 & 0,3 & 0,0 & 0,0 & 0,0 & 0,0 & 0,0 & 0,0 \\
\hline
\end{tabular}

A partir do levantamento feito no Diretório de Grupos de Pesquisa ${ }^{12}$ (CNPq, 2008), há evidências da concentração de certas áreas do conhecimento em determinadas questões ambientais. À exceção dos grupos de pesquisa da grande área "Linguística, Letras e Artes", que praticamente não apresentam afinidade com os temas ambientais selecionados para esta pesquisa, nota-se que o tema 12. Busca textual por "nome de grupo", "título da linha de pesquisa" e "palavras-chave da linha". ambiental mais presente entre os grupos de pesquisa registrados no CNPq é meio ambiente ( $\mathrm{N}=985$ em 2006 e $\mathrm{N}^{\prime}=1151$ em 2008). A base do CNPq pode apresentar um mesmo grupo de pesquisa relacionado a diferentes temas, o que resulta numa dupla contagem. Devido a esse fato, analisou-se o percentual de grupos de pesquisa que se vinculam às temáticas por grande área do conhecimento, a fim de observar a predominância temática entre elas. Os principais resultados da análise de dados indicam que:

1. Os temas desenvolvimento sustentável e meio ambiente apresentam uma concentração crescente de grupos de pesquisa das Ciências Sociais Aplicadas e das Ciências Humanas;

2. Para o tema recursos naturais, os grupos de pesquisa das Ciências Agrárias correspondem a cerca de um terço dos grupos ligados a esse tema; 
3. A biodiversidade é o foco privilegiado das Ciências Biológicas entre os temas pesquisados: seus grupos de pesquisa representam mais de dois terços de todos os grupos com esse foco temático;

4. Comparando os dados de 2006 e 2008 (Tabela 1) com os dados de Bursztyn (2004: 69), relativos à mesma base em 2002, destacam-se as seguintes alterações:

4.1. Houve um aumento no percentual de grupos de pesquisa que se referiram a recursos naturais nas Ciências Biológicas: de 7,06\% em 2002, para 16,04\% em 2006, e 16,08\% em 2008;

4.2. Houve um aumento no percentual de grupos de pesquisa da maioria das áreas do conhecimento pelo tema meio ambiente, exceto nas Ciências Biológicas (que representavam 10,27\% dos grupos interessados nesse tema em 2002 e passaram para 7,2\% em 2006, mantidos em 2008) e nas Ciências Exatas e da Terra, que representavam $20,54 \%$ dos grupos interessados nesse tema em 2002 e passaram para 16,44\% em 2006 e 15,46\% em 2008.

Assim, observam-se as diferenças entre as grandes áreas do conhecimento: ao se considerar as Humanidades (Ciências Humanas, Ciências Sociais Aplicadas e Linguística, Letras e Artes), evidencia-se a importância fundamental do tema desenvolvimento sustentável. Enquanto isso, biodiversidade e recursos naturais são temas enfocados preponderantemente por grupos das Ciências da Vida (Ciências Agrárias, Ciências da Saúde e Ciências Biológicas).

Esse conjunto de dados analisados reflete a crescente importância dos referidos temas para o desenvolvimento científico e tecnológico, ainda que as grandes áreas do conhecimento apresentem focos alternados nas pesquisas.

Isso significa que a interdisciplinaridade, enquanto processo e prática, cresce em importância, especialmente quando os problemas científicos se referirem à solução de problemas reais complexos, como é o caso das mudanças climáticas, das mutações genéticas, do ressurgimento de epidemias. As dimensões global e regional desses problemas geram diferentes incentivos para diversos atores cooperarem entre si (Dixit \& Skeath, 2001).

De fato, a prática interdisciplinar no Brasil tem encontrado obstáculos na alocação de investimentos públicos federais, na gestão e avaliação do processo de produção interdisciplinar de conhecimento científico e no próprio processo de pesquisa entre disciplinas sem a criação institucionalizada de programas "interdisciplinares" específicos - a rigidez institucional da organi- 
zação do conhecimento acadêmico em disciplinas dificulta soluções cooperativas para projetos de pesquisa entre grupos de diferentes departamentos ${ }^{13}$.

Problemas reais e complexos como esses exigem da comunidade científica novos parâmetros de divisão internacional do trabalho e de cooperação entre profissionais de diferentes formações e disciplinas no campo científico. Ou seja, que se produza de modo interdisciplinar novo conhecimento científico que responda às emergências e às demandas sociais do mundo em constante transformação.

\section{Considerações Finais}

Para os dois terços mais pobres da humanidade que vivem no Sul, o capital da natureza é sua fonte de manutenção e sustento. A destruição, o desvio e a ocupação de seus ecossistemas para extrair recursos naturais ou servir de depósito de lixo gera um fardo desproporcional para os pobres. Num mundo de comércio globalizado e desregulamentado, no qual tudo é negociável e a força econômica é o único determinante do poder e do controle, os recursos passam dos pobres para os ricos e a poluição, dos ricos para os pobres. O resultado é um apartheid ambiental global. (Shiva, 2004: 163-164)

Ao se observar novamente o cenário mundial, a consideração de Shiva (2004: 164) sobre a existência de um apartheid ambiental global parece enfraquecer a crença nos direitos humanos como algo pertencente a toda a humanidade. Isso se materializa, sobretudo, em certas ações de determinados organismos internacionais que fortalecem uma lógica econômica neoliberal baseada em imorais "janelas de oportunidade": altas taxas de mortalidade humana, legislações ambientais ineficientes, condições de trabalho irregulares e nações de miseráveis.

Em grande medida, as dificuldades impostas pela dinâmica contemporânea da nova produção de conhecimento científico e outras inerentes a ela, principalmente com relação a temas ambientais que necessitam de pesquisa interdisciplinar, reforçam tais condições de desigualdade. O presente artigo destacou um conjunto de fatores que permeia os cenários da prática interdisciplinar, a saber:

1. Na arena internacional, a importância da questão ambiental parece ser compartilhada por diversos discursos políticos (Bursztyn, 1995: 97), embora seja evidente que alguns países adotam estratégias diferentes de investimentos no desenvolvimento científico e tecnológico, produzido, portanto, de modo desigual e concentrado (Barros, 1999; 2005);

3. No Brasil, houve uma reorientação temática dos investimentos em C\&T, algo que poderia incrementar a prática de pesquisa interdisciplinar, mas
13. A literatura apresenta análises e estudos de casos específicos dentro de uma ou outra universidade ou centro de pesquisa (Epstein, 2003). Porém, há que se aprofundar as pesquisas nas bases CAPES e CNPq para poder analisar as relações entre grupos de diferentes departamentos em pesquisas conjuntas. 
que, de fato, ainda demonstra a dificuldade de superar a divisão de saberes disciplinares para pensar objetos complexos (Floriani, 2006; Baumgarten, 2006; Sobral, 2006a; Sobral 2006b; Bursztyn, 2004; 2005b).

Frente aos desafios impostos por diversas condições expostas anteriormente, conclui-se que as ciências sociais se sobressaem na construção dinâmica de um conhecimento interdisciplinar, sobretudo, porque oferecem diferentes teorias e métodos de análise e compreensão das questões subjetivas da realidade. É o novo espaço de um saber da práxis, um fazer-aprender-refazer, cujo motor depende da vontade, da "motivação" dos sujeitos de exteriorizarem suas disposições na prática produtiva que exercem em determinadas posições sociais (Bourdieu, 1994: 18).

De modo algum se excluem as Ciências Naturais dessa construção (Bursztyn \& Sayago, 2006: 104-106). Tendo a economia da inteligência como principal força produtiva no mundo contemporâneo (Gorz, 2005: 9), a construção de conhecimento é mais ou menos bem sucedida (aplicada a soluções de problemas sociais transformados em problemas científicos) quando houver maior ou menor ênfase na difícil prática interdisciplinar. Mas não se conhece tal prática de modo articulado em nível internacional. Enquanto isso, problemas reais permanecem sem solução:

Está ainda por avaliar cabalmente o impacto da biotecnologia agrícola na saúde ou no meio-ambiente. Se a produção pode aumentar exponencialmente, fa-lo-á à custa da biodiversidade. Se plantas e animais podem ser sujeitos à engenharia genética para se tornarem mais resistentes às doenças, à seca, ou aos herbicidas, isso é no fundo um incentivo a tolerar e até promover a degradação ecológica. (Santos, 1999: 292)

Apesar da prática de pesquisa interdisciplinar no cenário mundial não estar sistematizada e das dificuldades relativas à institucionalização de cursos interdisciplinares, observou-se que, no Brasil, todas as áreas do conhecimento possuem grupos de pesquisa que trabalham temas predominantes na questão ambiental, num envolvimento crescente e constante. Todavia, as Ciências Humanas e as Ciências Sociais Aplicadas concentram-se em pesquisas sobre meio ambiente e desenvolvimento sustentável, enquanto as Biológicas, Agrárias e Exatas dedicam-se majoritariamente às temáticas da biodiversidade e dos recursos naturais.

Desse modo, os resultados analisados dessa prática interdisciplinar indicam um potencial de complementaridade entre essas áreas do conhecimento com relação à questão ambiental. Portanto, o principal desafio consiste nos modos de relacionamento e ação coletiva, que envolvem coordenação, cooperação e confiança entre cientistas de diferentes áreas na resolução de problemas complexos que compartilham, bem como formas de se garantir fontes de 
financiamento sistemático e consistente com a dimensão do que se pesquisa.

Finalmente, o que se pode inferir dos dados e das teorias apresentados anteriormente é que, mesmo numa contemporaneidade complexa, capaz de replicar (e provavelmente redesenhar) códigos genéticos das diversas formas de vida, ainda existem limites à ação humana e consequências nefastas desta sobre a natureza. Caso não sejam construídos coletivamente movimentos articulados nacionais e internacionais em prol de uma ecologia humana (Latour, 2004), que passem por uma tomada de consciência humana para si, não se desenvolverão soluções exequíveis e sustentáveis a longo prazo.

E, sem uma percepção unificada com base em legislação e regulação internacionais (Altvater, 1999: 147), capazes de orientar e fiscalizar políticas científicas e ambientais cooperativas e mundializadas, as forças desmedidas e avassaladoras da Natureza poderão nos impingir a morte, enfim.

\section{Referências}

Altvater, E. (1999) "Os desafios da globalização e da crise ecológica para o discurso da democracia e dos direitos humanos", In Heller, A. et al. A crise dos paradigmas em ciências sociais e os desafios para o século XXI. Rio de Janeiro, Contraponto.

BarRos, F. A. F. (1999). Confrontos e contrastes regionais da ciência e tecnologia no Brasil. Brasília, UNB/ Paralelo 15.

- (2005) A tendência concentradora da produção do conhecimento no mundo contemporâneo. Brasília, Paralelo 15.

BARTHOLO JR., R. S. (1984) “A Crise do Industrialismo: genealogia, riscos e oportunidades", In BURSZTYN et al (orgs.), Que Crise é essa? São Paulo, Brasiliense: 69-101.

Baumgarten, M. (2006) "Sociedade e conhecimento - ordem, caos e complexidade" (Apresentação). Sociologias, ano 8, n. 15: 16-23.

BECK, U. (2002) La Sociedad del Riesgo Global. Madri, Siglo Veintiuno.

BouRdieu, P. (1994) Razões Práticas: sobre a teoria da ação. Campinas, Papirus. . (2001) Science de la Science et Réflexivité. Paris, Éditions Raisons d'Agir. . (2003) Os Usos Sociais da Ciência. São Paulo, Editora UNESP. . (2004) A Economia das Trocas Simbólicas. 5a. ed. São Paulo, Perspectiva. 
BoURdieU, P. \& WACQUANT, L. (1992). Réponses pour une anthropologie réflexive. Paris, Ed. Du Seuil.

BURSZTYN, M. (1995). "Armadilhas do progresso: contradições entre economia e ecologia". Sociedade e Estado, Vol. X, n. 1, Jan-Jun: 97-124.

. (2004) "Meio Ambiente e Interdisciplinaridade - desafios ao mundo acadêmico". Desenvolvimento e Meio Ambiente, Vol. 10, n. 1: 67-76.

. (2005a) A Difícil Sustentabilidade: política energética e conflitos socioambientais. Rio de Janeiro, Garamond.

. (2005b) "A Institucionalização da Interdisciplinaridade na Universidade Brasileira". Revista Liinc, Vol. 1, n.0: 38-52.

BuRSzTYN, M. \& SAYAGO, D. (2006) "A tradição da ciência e a ciência da tradição: relações entre valor, conhecimento e ambiente", In Garay, I. \& Becker, B. Dimensões Humanas da Biodiversidade. Petrópolis, Vozes.

Castells, M. (2005). A Sociedade em Rede, Vol. I. 8a ed. São Paulo, Paz e Terra.

Coordenação De Aperfeiçoamento De Pessoal De Nivel Superior - CAPES (2008). "Cursos recomendados", In http://www.capes.gov.br/avaliacao/ recomendados.html

. (2008b). "Relação de Cursos Recomendados e Reconhecidos", Grande Área Multidisciplinar, Área Interdisciplinar, In http://conteudoweb.capes.gov. $\mathrm{br} /$ conteudoweb/ProjetoRelacaoCursosServlet?acao=pesquisarles\&codigoArea $=90100000 \&$ descricaoArea $=$ MULTIDISCIPLINAR+\&descricaoAreaConhecimento =INTERDISCIPLINAR\&descricaoAreaAvaliacao=MULTIDISCIPLINAR

Conselho Nacional De Desenvolvimento Cientifico E Tecnológico - CNPQ. (2008). Diretório de Grupos de Pesquisa Lattes, In http://dgp.cnpq.br/censos/

Dixit, A. \& SkEATH, S. (1999) Games of Strategy. New York, W.W. Norton \& Company.

ELIAS, N. (1994a) A Sociedade dos Indivíduos. Rio de Janeiro, Jorge Zahar. . (1994b) O Processo Civilizador. Vol. I. Rio de Janeiro, Jorge Zahar.

FazendA, I. C. A. (1994) Interdisciplinaridade: história, teoria e pesquisa. Campinas, Papirus.

FLoRIANI, D. (2006) “Ciências em Trânsito, Objetos Complexos: práticas e discursos socioambientais". Ambiente e Sociedade, Vol. IX, n. 1, jan/jun: 65-80.

FURTADO, C. (1996) O Mito do Desenvolvimento Econômico. (Coleção Cultura). Rio de Janeiro, Paz e Terra. 
GibBons, M. et al. (1993) The New Production of Knowledge: the dynamics of science and research in contemporary societies. London, SAGE.

Giddens, A., Beck, U. \& LASH, S. (1997) Modernização Reflexiva, São Paulo, Editora da UNESP.

Gorbachev, M. (2003) Meu Manifesto pela Terra. São Paulo, Planeta do Brasil.

Gorz, A. (2005) O Imaterial. São Paulo, Anna Blume.

Habermas, J. (1975) "Técnica e Ciência Enquanto Ideologia", In Os pensadores, Vol. XLVIII. São Paulo, Abril Cultural.

Herrera, A. (1984) "A Crise da Espécie”, In Bursztyn, M., Leitão, P. \& Chain, A. (orgs.), Que Crise é Essa? São Paulo, Brasiliense/ CNPq.

KnoRR-CetinA, K. (1982) "Scientific communities or transepistemic arenas of research? A critique of quasi economic models of science". Social Studies of Science, n. 12: 101-130.

LAtour, B. (2000) Ciência em ação: como seguir cientistas e engenheiros sociedade afora. São Paulo, UNESP. . (2002) War of the Worlds: what about peace? Chicago, Prickly Paradigm. - (2004) Políticas da Natureza. São Paulo, EDUSC.

LÉlÉ, S. \& NorgaArd, R. (2005). "Practicing Interdisciplinary". Bioscience, Vol. 55, n. 11: 967-975.

LONG, B. (2000) International Environmental issues and the OECD: an historical perspective. Paris, OEDC.

Maranhão, T. de P. A. (2005) A Autonomia Reflexiva na Ciência Brasileira. Mimeografado. Brasília, Departamento de Sociologia/ UnB.

MARcuse, H. (1967) A Ideologia da sociedade industrial. Rio de Janeiro, Zahar.

Martins, C. B. (2000) "O ensino superior brasileiro nos anos 90". São Paulo Perspectivas, Vol. 14, n. 1: 41-60.

Ministérıo Da CıênCIA E TeCnologia. (2001) Livro Verde. Brasília, MCT.

MorIn, E. (1993) Terre Patrie. Paris, Ed. Du Seuil.

Nelissen, N. et al. (edits.) (1997) Classics in Environmental Studies. Utrecht, International Books. 
Nowotny, H., Scott, P. \& GibBons, M. (2001) Re-Thinking Science: knowledge and the public in an age of uncertainty. Cambridge, Polity Press.

SACHS, I. (2004) Desenvolvimento: includente, sustentável, sustentado. Rio de Janeiro, Garamond.

Santos, B. de S. (1999) Pela Mão de Alice: o social e o político na pós-modernidade. São Paulo, Cortez.

ShIVA, V. (2004) "O Mundo no Limite", In Hutton, W. \& Giddens, A. (orgs.), No Limite da Racionalidade: convivendo com o capitalismo global. Rio de Janeiro, Record: 163-186.

Sobral, F. A. da F. (2004) "O Modelo Misto de Desenvolvimento Científico e Tecnológico no Fomento à Pesquisa Brasileira", In http://www.ces.uc.pt/ lab2004/inscricao/pdfs/painel44/FernandaSobral.pdf

. (2006a) "Fundos Setoriais e Interdisciplinaridade", paper apresentado no ST08- Redes, Conhecimentos e Inovação Social, no 30 Encontro Anual da ANPOCS, 24 a 28 de outubro. Caxambu.

. (2006b) "A Pós-Graduação Brasileira", In Porto, M. S. G. \& Dwyer, T. (orgs.), Sociologia e Realidade: pesquisa social no século XXI. Brasília, Universidade de Brasília: 157-171.

. \& Maranhão, T. de P. A. (2008) “La Autonomía Reflexiva en el Campo de la Sociología (2000-2006)". Íconos. Revista de Ciencias Sociales, n. 31.

Sobral, F. \& TRIGUeIRo, M. (1994) "Limites e potencialidades da base técnicocientifica", In Fernandes, A. M. \& Sobral, F. (orgs.), Colapso da Ciência \& Tecnologia no Brasil. Rio de Janeiro, Relume- Dumará: 71 - 100.

TeIXEIRA, O. A. (2004) "Interdisciplinaridade: problemas e desafios", In Revista Brasileira de Pós-Graduação, n. 1: 57-69.

VEIGA, J. E. da (2005) Desenvolvimento Sustentável: desafio do século XXI. Rio de Janeiro, Garamond.

VIOLA, E. et al. (1998) Meio Ambiente, Desenvolvimento e Cidadania: desafio para as ciências sociais. São Paulo, Cortez.

Weber, M. (1967) A Ética Protestante e o Espírito do Capitalismo. São Paulo, Pioneira.

Zellmer, A. J, Allen, T.F.H. \& Kessenboehmer, K. (2006) "The Nature of Ecological Complexity: a protocol for building the narrative" (viewpoint). Ecological Complexity, vol. 3: 171-182. 